\title{
Ageing-associated changes in the human DNA methylome: genomic locations and effects on gene expression
}

Saara Marttila ${ }^{1,2^{*}}$, Laura Kananen ${ }^{1,2}$, Sergei Häyrynen ${ }^{3}$, Juulia Jylhävä ${ }^{1,2}$, Tapio Nevalainen ${ }^{1,2}$, Antti Hervonen ${ }^{2,4}$, Marja Jylhä̈ ${ }^{2,4}$, Matti Nykter ${ }^{3}$ and Mikko Hurme $e^{1,2,5}$

\begin{abstract}
Background: Changes in DNA methylation are among the mechanisms contributing to the ageing process. We sought to identify ageing-associated DNA methylation changes at single-CpG-site resolution in blood leukocytes and to ensure that the observed changes were not due to differences in the proportions of leukocytes. The association between DNA methylation changes and gene expression levels was also investigated in the same individuals.

Results: We identified 8540 high-confidence ageing-associated CpG sites, 46\% of which were hypermethylated in nonagenarians. The hypermethylation-associated genes belonged to a common category: they were predicted to be regulated by a common group of transcription factors and were enriched in a related set of GO terms and canonical pathways. Conversely, for the hypomethylation-associated genes only a limited set of GO terms and canonical pathways were identified. Among the $8540 \mathrm{CpG}$ sites associated with ageing, methylation level of 377 sites was also associated with gene expression levels. These genes were enriched in GO terms and canonical pathways associated with immune system functions, particularly phagocytosis.

Conclusions: We find that certain ageing-associated immune-system impairments may be mediated via changes in DNA methylation. The results also imply that ageing-associated hypo- and hypermethylation are distinct processes: hypermethylation could be caused by programmed changes, whereas hypomethylation could be the result of environmental and stochastic processes.
\end{abstract}

Keywords: Epigenetics, Methylome, DNA methylation, Ageing, PBMCs, Gene expression, Molecular ageing, Hypermethylation, Hypomethylation

\section{Background}

Ageing can be described as a functional decline that leads to a diminished ability to respond to stress, increased homeostatic instability and an increased risk of diseases such as cancer and inflammatory diseases. Ultimately, these changes lead to death [1]. The molecular basis of ageing is multifactorial, including changes in energy metabolism, alterations in DNA repair mechanisms, increased inflammation and changes in leukocyte proportions (changes in $\mathrm{CD} 4+/ \mathrm{CD} 8+$ ratio, increase of costimulatory CD28 receptordeficient $\mathrm{T}$ cells [2]). Consequently, several theories exist

\footnotetext{
*Correspondence: saara.marttila@uta.fi

'Department of Microbiology and Immunology, School of Medicine, University of Tampere, Tampere, Finland

${ }^{2}$ Gerontology Research Center, Tampere, Finland

Full list of author information is available at the end of the article
}

regarding the mechanisms underlying ageing. Whether the ageing process itself consists of the accumulation of molecular damage due to environmental and stochastic effects or is a truly programmed or pseudo-programmed process that stems from development remains to be determined, yet a process as complex as ageing most likely involves aspects of all these phenomena [3-5].

Ageing leads to both global and local changes in the DNA methylation profile. Global hypomethylation has been shown to occur across tissues, and promoter-specific hypermethylation has been demonstrated for various tissues and genes [6]. Several ageing-relates diseases, such as cancer, Alzheimer's disease and type 2 diabetes, have also been shown to be associated with changes in DNA methylation [7]. The role of epigenetics in ageing-associated processes 
could be significant, as genetics appears to explain only a small portion of the observed variation in lifespan and healthspan [8]. As the epigenome is modified throughout life by varying environmental conditions, the accumulated effects of these changes could be most prominent in the aged population.

DNA methylation was suggested to control the activity of genes as early as $1975[9,10]$ and has since been demonstrated to control the expression of single genes and the silencing of large sections of chromatin. DNA methylation mainly occurs on CpG-dinucleotides, which form $\mathrm{CpG}$ islands containing above-average $\mathrm{CpG}$ content. These $\mathrm{CpG}$ islands overlap the transcription start sites (TSSs) of the majority of human genes, and the classical role of DNA methylation is transcriptional inhibition, with the methylation of TSSs preventing the initiation of transcription $[11,12]$. The role of methylation in the gene body is less clear; methylation does not appear to block transcriptional elongation but may actually enhance it, and methylation may have a role in alternative splicing. Furthermore, DNA methylation is required for the suppression of transposable elements [13]. DNA methylation controls gene expression by directly inhibiting the binding of transcription factors (TFs), by recruiting methyl-binding proteins that prevent TFs from binding to DNA [14], or by affecting the conformation of the surrounding chromatin [15].

The relationship between ageing and DNA methylation has been studied previously by measuring the DNA methylation level of repetitive elements (global DNA methylation [6]) as well as with Illumina Golden Gate array [16] and the Infinium HumanMethylation27 BeadChip (27 K array) [17-22]. These arrays included a severely biased set of CpGs located in known cancer-associated genes and CpGs located almost exclusively in CpG island promoter regions, respectively. The Illumina Infinium HumanMethylation450 BeadChip (450K array) offers an improvement in this area, as the probes span $99 \%$ of the RefSeq genes and are distributed more evenly across the genome, such as on the shores and shelves of CpG islands and in non-CpG islands (non-CGIs), as well as in gene bodies and untranslated regions (UTRs) [23-28]. However, the majority of previous studies did not take into consideration the prominent ageing-associated changes in the proportions of leukocytes, thereby introducing possible bias into the analyses [29].

In this study, our aim was to identify ageing-associated DNA methylation changes that are independent of changes in leukocyte proportions. We also examined gene expression data from the same individuals from whom the methylation data were obtained, and we were therefore able to explore the relationship between gene expression and DNA methylation in these elderly individuals.

\section{Results}

\section{Ageing-associated DNA methylation changes}

Our study population consisted of the Vitality $90+$ study population: there was a total of 146 nonagenarians and 30 young controls, from whom we extracted peripheral blood mononuclear cells (PBMCs). The methylation data were produced with the $450 \mathrm{~K}$ array, and the expression data were obtained with the Illumina HumanHT12v4 BeadChip. Our aim was to identify ageing-associated changes in the level of DNA methylation. Our approach was two-sided, as we sought to concentrate on $\mathrm{CpG}$ sites that showed a large enough difference in the level of methylation to have a plausible biological significance but also to ensure that the identified differences were not due to changes in the proportions of leukocyte populations.

The proportions of different leukocytes differed between the nonagenarians and young controls in our study population, as reported previously [30]. A principal component analysis (PCA) revealed that the first principal component accounted for $20.5 \%$ of the observed variation in methylation levels detected in our data (Figure 1). This component was strongly associated with leukocyte proportions, indicating that the analysis needs to be adjusted for the proportions of leukocytes.

First, we compared the methylation levels at individual CpGs in the nonagenarian group $(n=122)$ with those in the young control group $(n=21)$ using the Wilcoxon rank-sum test and identified $10083 \mathrm{CpG}$ sites that were differentially methylated between these two groups (with a Benjamini-Hochberg-corrected $\mathrm{p}$-value $<0.05$ and a difference between absolute $\mathrm{M}$-value medians $>1$ ). Second, age group-associated methylation sites were identified with a beta regression model, with sex and different leukocyte populations (the ratio of $\mathrm{CD} 4+$ and $\mathrm{CD} 8+\mathrm{T}$ cells and the proportions of CD4 + CD28-, CD8 + CD28and CD14+ cells) as covariates. This method identified $45507 \mathrm{CpG}$ sites for which age group was a significant covariate (Bonferroni-corrected p-value <0.05). The 10083 CpG sites identified via the group comparison were enriched at the top of the list of the 45507 ageing-associated CpGs. However, 1543 of the 10083 CpG sites showed no statistical significance in the regression analysis, indicating that the perceived difference in methylation was due to differences in leukocyte proportions rather than ageing per se. We now report the $8540 \mathrm{CpG}$ sites, which exhibit a large, statistically significant difference in the level of methylation between the nonagenarians and young controls and remain significant after adjusting for differences in leukocyte populations in the regression analysis, as truly ageing-associated methylation changes (for a list of all ageing-associated CpGs, see Additional file 1). Sex chromosomes were excluded from the analysis. 


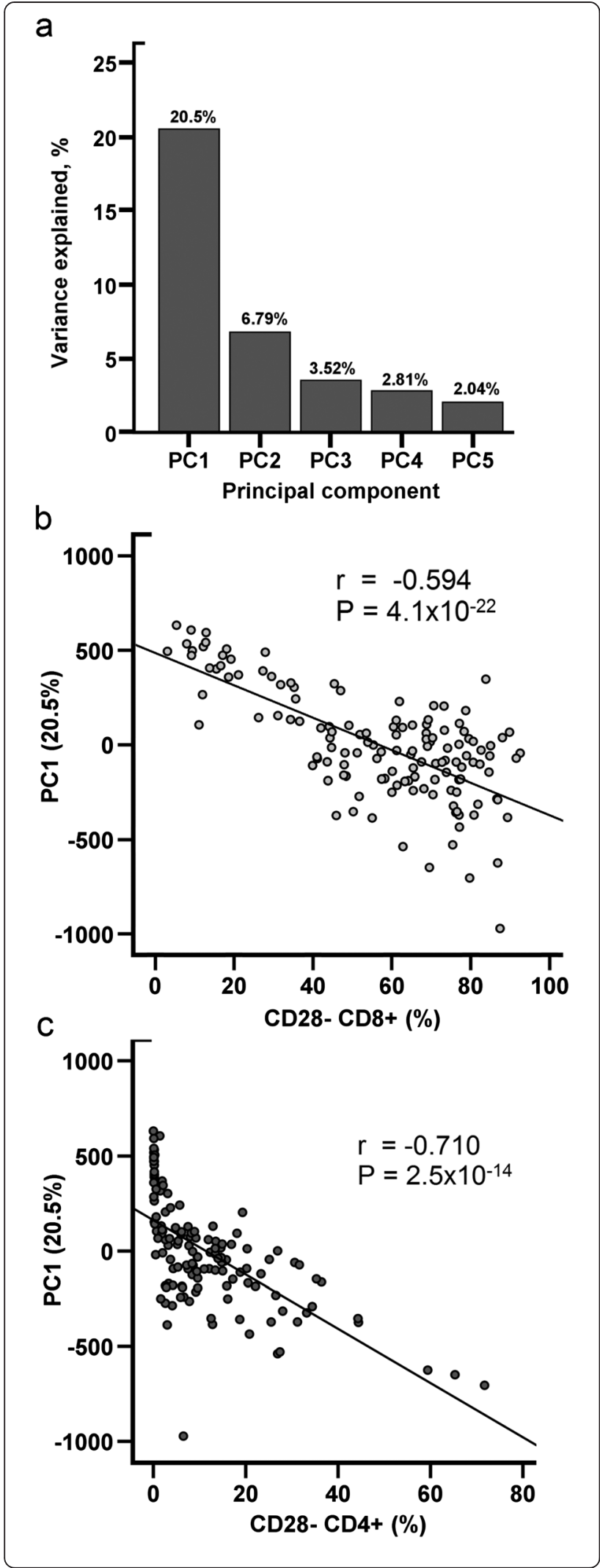

Figure 1 The association of cell type proportions with DNA methylation. The global DNA methylation was decomposed into a set of linearly independent principal component (PC) patterns. Components were used to examine the relationships between global DNA methylation and biological or non-biological covariates (e.g., gender, the batch effect and cell types). (a) The top 5 components (PC1-PC5) with the largest proportion of explained variance from the data. The percentages of explained variance are shown above the bars. (b) The association of the proportion of CD8 + CD28- cells with the first principal component (Spearman's rank correlation coefficient -0.594 ( $p=4.1 \mathrm{e}-22)$ ) and (c) the association of the proportion of CD4 + CD28- cells with the first principal component (Spearman's rank correlation coefficient -0.710 $(p=2.5 e-14))$.

Among the 8540 ageing-associated CpG sites, 3925 (46\%) were hypermethylated, while 4615 (54\%) were hypomethylated, in the nonagenarians. The most significant hits, based on the p-values obtained using the sitespecific regression models, were cg16867657 (ELOVL2), cg16762684 (MBP), cg11344352 (ERCC1), cg17110586 and $\operatorname{cg} 04875128$ (OTUD7A). The largest differences in the level of methylation were observed for cg07211259 (PDCD1LG2), cg18826637 and cg26063719 (VIM), which were hypomethylated in the nonagenarians, and for cg06352730, cg00674365 (ZNF471) and cg21402921 (GABRA5), which were hypermethylated in the nonagenarians. The top-ranking hits are presented in Tables 1 , 2 and Figure 2.

\section{Genomic location of the ageing-associated methylation} sites

The ageing-associated CpGs were not uniformly distributed across chromosomes, CpG islands or genes. Chromosomes 2, 3, 4, 5 and 18 contained more ageingassociated methylation sites than expected, whereas chromosomes 16, 17, 19 and 22 had fewer ageing-associated methylation sites than expected (Hypergeometric test $\mathrm{p}<0.05$, Additional file 2). On the majority of these chromosomes, the proportion of hypermethylated sites compared with the proportion of hypomethylated sites was roughly equal or was slightly biased towards an excess of hypomethylated sites, as in the overall data. Interestingly, on chromosomes 18 and 19, there were considerably more hypermethylated sites than expected: among the identified ageing-associated methylation sites on these chromosomes, $72 \%$ and $75 \%$ were hypermethylated, constituting a clear overrepresentation compared with the $46 \%$ of hypermethylated sites identified in the total data.

The CpG sites that were hypermethylated with advancing age were enriched at $\mathrm{CpG}$ islands, rather than on island shores or shelves or in non-CGIs. By contrast, the hypomethylated CpG sites were enriched in non-CGIs; their absence from $\mathrm{CpG}$ islands was striking, as only $1.2 \%$ of all hypomethylated sites were located in CpG islands, whereas $31.5 \%$ of the total probes were located 
Table 1 Top 10 age-group associated CpG sites from the regression model

\begin{tabular}{|c|c|c|c|c|c|}
\hline ProbelD & Gene & betareg estimate & betareg $p$-value & $\Delta \beta$ & Wilcoxon p-value \\
\hline $\operatorname{cg} 16867657$ & ELOVL2 & 1.023 & $6.38 \mathrm{E}-66$ & 0.243 & $1.53 \mathrm{E}-10$ \\
\hline $\operatorname{cg} 16762684$ & $M B P$ & -1.486 & $4.74 \mathrm{E}-64$ & -0.168 & $1.53 \mathrm{E}-10$ \\
\hline $\operatorname{cg} 11344352$ & ERCC1 & -1.202 & $9.15 \mathrm{E}-63$ & -0.153 & $1.53 \mathrm{E}-10$ \\
\hline $\operatorname{cg} 17110586$ & na & 0.895 & $1.46 \mathrm{E}-59$ & 0.200 & $1.53 \mathrm{E}-10$ \\
\hline cg04875128 & OTUDTA & 1.514 & $7.2 \mathrm{E}-58$ & 0.279 & $1.53 \mathrm{E}-10$ \\
\hline cg08262002 & $\angle D B 2$ & -0.710 & $2.72 \mathrm{E}-55$ & -0.197 & $1.53 \mathrm{E}-10$ \\
\hline cg18618815 & COLIAI & -0.941 & $1.78 \mathrm{E}-52$ & -0.225 & $1.53 \mathrm{E}-10$ \\
\hline cg00748589 & na & 0.864 & $1.36 \mathrm{E}-51$ & 0.179 & $1.53 \mathrm{E}-10$ \\
\hline cg15416179 & MAP2K3 & -1.131 & $2.38 \mathrm{E}-51$ & -0.187 & $1.53 \mathrm{E}-10$ \\
\hline cg12065799 & RRAGC & -0.823 & $8.15 E-51$ & -0.088 & $1.53 \mathrm{E}-10$ \\
\hline cg23479922 & MARCH11 & 0.940 & 4.07E-49 & 0.263 & $1.53 \mathrm{E}-10$ \\
\hline cg07544187 & CILP2 & 1.541 & $2.35 \mathrm{E}-48$ & 0.252 & $1.53 \mathrm{E}-10$ \\
\hline cg09038267 & C10orf26 & 1.227 & $1.48 \mathrm{E}-47$ & 0.150 & $1.53 \mathrm{E}-10$ \\
\hline $\operatorname{cg} 13033938$ & IP6K1 & -0.699 & $7.54 \mathrm{E}-47$ & -0.061 & $1.53 \mathrm{E}-10$ \\
\hline cg19283806 & CCDC102B & -1.253 & $9.82 \mathrm{E}-47$ & -0.267 & $1.53 \mathrm{E}-10$ \\
\hline cg07547549 & SLC12A5 & 0.900 & $5.02 \mathrm{E}-46$ & 0.245 & $1.53 \mathrm{E}-10$ \\
\hline cg01949403 & APOL3 & 0.807 & $7.53 \mathrm{E}-46$ & 0.111 & $1.53 \mathrm{E}-10$ \\
\hline cg01243823 & NOD2 & -1.280 & $7.9 \mathrm{E}-46$ & -0.232 & $1.53 \mathrm{E}-10$ \\
\hline cg22242842 & na & -0.952 & $1.99 \mathrm{E}-44$ & -0.206 & $1.53 \mathrm{E}-10$ \\
\hline cg06007201 & FAM38A & -0.932 & $5.65 \mathrm{E}-44$ & -0.156 & $1.53 \mathrm{E}-10$ \\
\hline
\end{tabular}

CpG sites with most significant association to age group in the beta regression models (betareg). To clarify, $\Delta \beta$ refers to difference in the median of DNA methylation values between nonagenarians and young controls (difference in $\beta$-value), whereas betareg estimate refers to the estimate obtained from a regression model termed beta regression. Thus the absolute value of betareg estimate and the absolute value of $\Delta \beta$ for a given CpG site are not directly comparable, only the signs of the values are.

in $\mathrm{CpG}$ islands (Figure 3). In regard to gene regions, hypermethylated CpGs were enriched in regions near the TSSs and the $1^{\text {st }}$ exons of genes, whereas hypomethylated sites were scarce in these areas and were enriched in the gene body and, more strongly, in the regions outside of genes (Figure 3).

\section{Functional annotation of the ageing-associated methylation sites}

The locations of ageing-associated hyper- and hypomethylation differ, thus it can be assumed that their origins and/or functions also differ. Therefore, we performed the functional analyses separately for hypermethylated and hypomethylated sites and genes harbouring these ageingassociated methylation sites. The 3925 hypermethylated sites were annotated to 1832 different genes, and the 4615 hypomethylated CpG sites were annotated to 2057 different genes.

GOrilla (Gene Ontology enRIchment anaLysis and visuaLizAtion tool) $[31,32]$ was used to identify the GO functions and processes associated with ageing-associated hyper- and hypomethylation-associated genes. For both categories, we identified more significant GO terms for hypermethylation-associated genes, even though there were fewer hypermethylation-associated genes compared with hypomethylation-associated genes. For the hypermethylation-associated genes, 36 enriched GO function terms were identified (Bonferroni corrected $\mathrm{p}<0.05$ ), whereas for the hypomethylation-associated genes, 27 enriched GO function terms were identified; 11 of these terms were common to the two groups (Additional file 3). The top GO function terms for the hypermethylation-associated genes were unique to these genes; these terms were associated with sequence-specific DNA binding and transcription factor binding (also presented as a diagram in Additional file 4). The GO terms that were enriched only for hypomethylated sites did not reveal similar enrichment for a common process (Additional file 4). The GO function terms that were common to hypermethylation- and hypomethylation-associated genes also formed a group and were clustered around channel function-associated GO terms. The results for GO process terms was similar to that for GO functions, as we identified 265 significant GO terms for hypermethylation-associated genes, whereas for hypomethylation-associated genes, we identified only 53 significant GO terms; 41 of these terms were common to hyper- and hypomethylation-associated genes. (Additional file 5). The top-ranking hypermethylation- 
Table 2 Top $10 \mathrm{CpG}$ sites with the largest $\Delta \beta$ between nonagenarians and young controls

\begin{tabular}{llllll}
\hline ProbelD & Gene & $\begin{array}{l}\text { betareg } \\
\text { estimate }\end{array}$ & $\begin{array}{l}\text { betareg } \\
\text { p-value }\end{array}$ & $\boldsymbol{\Delta} \boldsymbol{\beta}$ & $\begin{array}{l}\text { Wilcoxon } \\
\text { p-value }\end{array}$ \\
\hline cg07211259 & PDCD1LG2 & -1.086 & $3.24 \mathrm{E}-30$ & -0.290 & $1.53 \mathrm{E}-10$ \\
cg18826637 & na & -1.280 & $2.23 \mathrm{E}-32$ & -0.289 & $1.53 \mathrm{E}-10$ \\
cg26063719 & VIM & -1.036 & $6.09 \mathrm{E}-25$ & -0.284 & $1.53 \mathrm{E}-10$ \\
cg08548498 & SLPI & -0.767 & $1.24 \mathrm{E}-15$ & -0.278 & $1.66 \mathrm{E}-10$ \\
cg19283806 & CCDC102B & -1.253 & $9.82 \mathrm{E}-47$ & -0.267 & $1.53 \mathrm{E}-10$ \\
cg13591783 & ANXA1 & -0.826 & $5.17 \mathrm{E}-22$ & -0.266 & $1.53 \mathrm{E}-10$ \\
cg27192248 & na & -1.246 & $2.57 \mathrm{E}-20$ & -0.265 & $1.59 \mathrm{E}-10$ \\
cg03274391 & na & -1.263 & $1.18 \mathrm{E}-15$ & -0.264 & $1.54 \mathrm{E}-10$ \\
cg23654401 & VOPP1 & -0.781 & $2.94 \mathrm{E}-16$ & -0.262 & $1.54 \mathrm{E}-10$ \\
cg26269881 & BHLHE40 & -1.005 & $4.25 \mathrm{E}-25$ & -0.261 & $1.53 \mathrm{E}-10$ \\
cg18952796 & NPTX2 & 1.121 & $6.89 \mathrm{E}-26$ & 0.264 & $1.56 \mathrm{E}-10$ \\
cg17688525 & L3MBTL4 & 0.865 & $1.36 \mathrm{E}-11$ & 0.265 & $5.86 \mathrm{E}-10$ \\
cg27526665 & THRB & 0.940 & $2.64 \mathrm{E}-22$ & 0.266 & $2.0 \mathrm{E}-10$ \\
cg09555124 & IGF2R & 0.944 & $4.34 \mathrm{E}-23$ & 0.277 & $1.53 \mathrm{E}-10$ \\
cg23160016 & GABRA2 & 1.041 & $1.01 \mathrm{E}-17$ & 0.277 & $2.49 \mathrm{E}-10$ \\
cg10568066 & RNF39 & 0.973 & $4.68 \mathrm{E}-13$ & 0.278 & $1.60 \mathrm{E}-8$ \\
cg04875128 & OTUD7A & 1.514 & $7.2 \mathrm{E}-58$ & 0.279 & $1.53 \mathrm{E}-10$ \\
cg21402921 & GABRA5 & 0.868 & $4.90 \mathrm{E}-17$ & 0.285 & $5.58 \mathrm{E}-10$ \\
cg00674365 & ZNF471 & 1.033 & $6.27 \mathrm{E}-24$ & 0.288 & $3.65 \mathrm{E}-10$ \\
cg06352730 & na & 1.437 & $1.26 \mathrm{E}-23$ & 0.288 & $1.76 \mathrm{E}-10$ \\
\hline CpG 105 wh & & & &
\end{tabular}

CpG sites with the largest difference in the methylation level $(\Delta \beta)$ between nonagenarians and controls. To clarify, $\Delta \beta$ refers to difference in the median of DNA methylation values between nonagenarians and young controls (difference in $\beta$-value), whereas betareg estimate refers to the estimate obtained from a regression model termed beta regression. Thus the absolute value of betareg estimate and the absolute value of $\Delta \beta$ for a given $\mathrm{CpG}$ site are not directly comparable, only the signs of the values are. specific GO terms were clustered around two types of processes: development and morphogenesis; and metabolic processes, gene expression and nucleotide metabolism (Additional file 6). Again, the significant GO terms associated with hypomethylation did not belong to a specific group (Additional file 6). The hypermethylation-specific GO terms that formed specific clusters are presented in Tables 3, 4 and 5 .

PScan [33] was used to identify transcription factors that could be common regulators of the identified genes. For the hypermethylation-associated genes, 24 common transcription factors were identified (Additional file 7), whereas for the hypomethylation-associated genes, only one TF (EWSR1-FLI1, p-value 1.502e-5), was identified. Among the 24 transcription factors that were common to hypermethylation-associated genes, half (12) were zinc-coordinating transcription factors.

We also identified canonical pathways related to hypoand hypermethylation-associated genes through Ingenuity pathway analysis (IPA) [34]. For the hypermethylationassociated genes, we identified 19 affected canonical pathways (Benjamini-Hochberg-corrected p-value <0.05), whereas for the hypomethylation-associated genes, 3 pathways were identified, 1 of which was common to both groups of genes (Additional file 8). The canonical pathways associated with hypermethylation in nonagenarians belonged to signalling pathway categories such as Organismal growth $\mathcal{E}$ development, Cellular growth and Proliferation and development (Figure 4).

\section{Effect of sex on ageing-associated DNA methylation changes}

Among the 8540 ageing-associated, high-confidence CpG sites, only 7 showed a statistically significant association with sex in our beta regression analysis in which age group, sex and leukocyte proportions were included

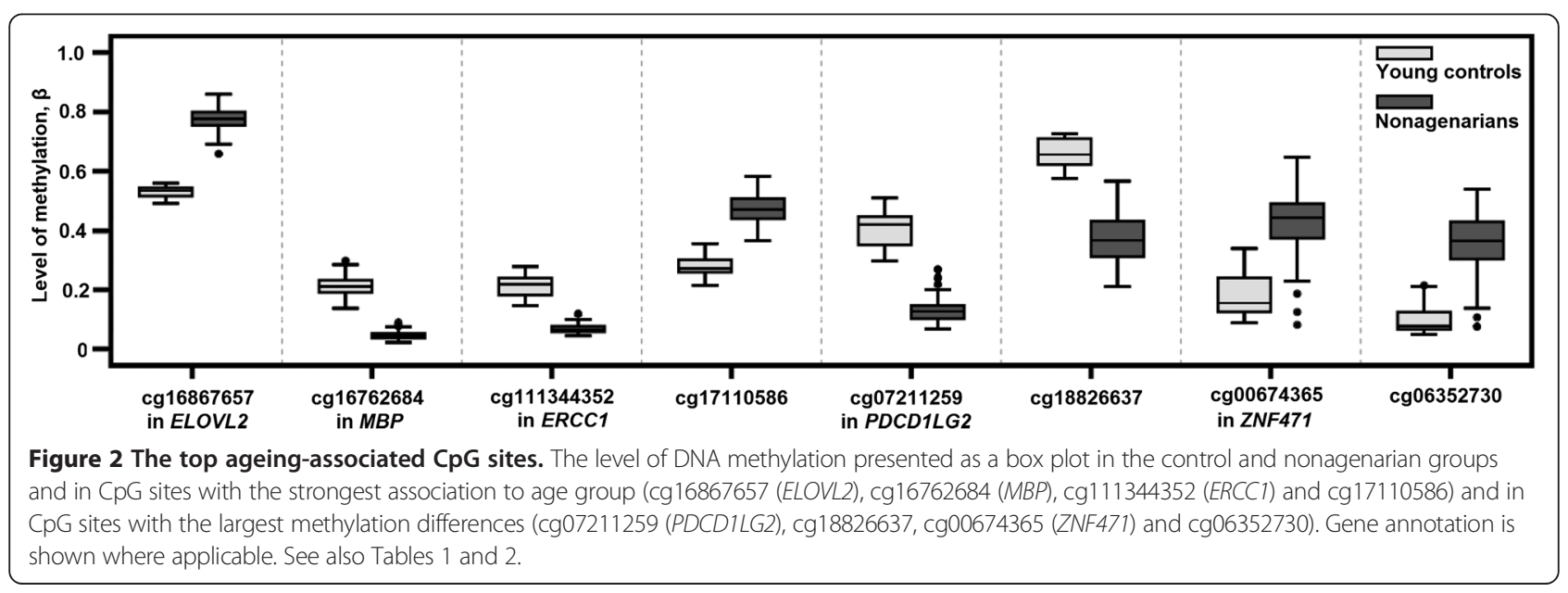




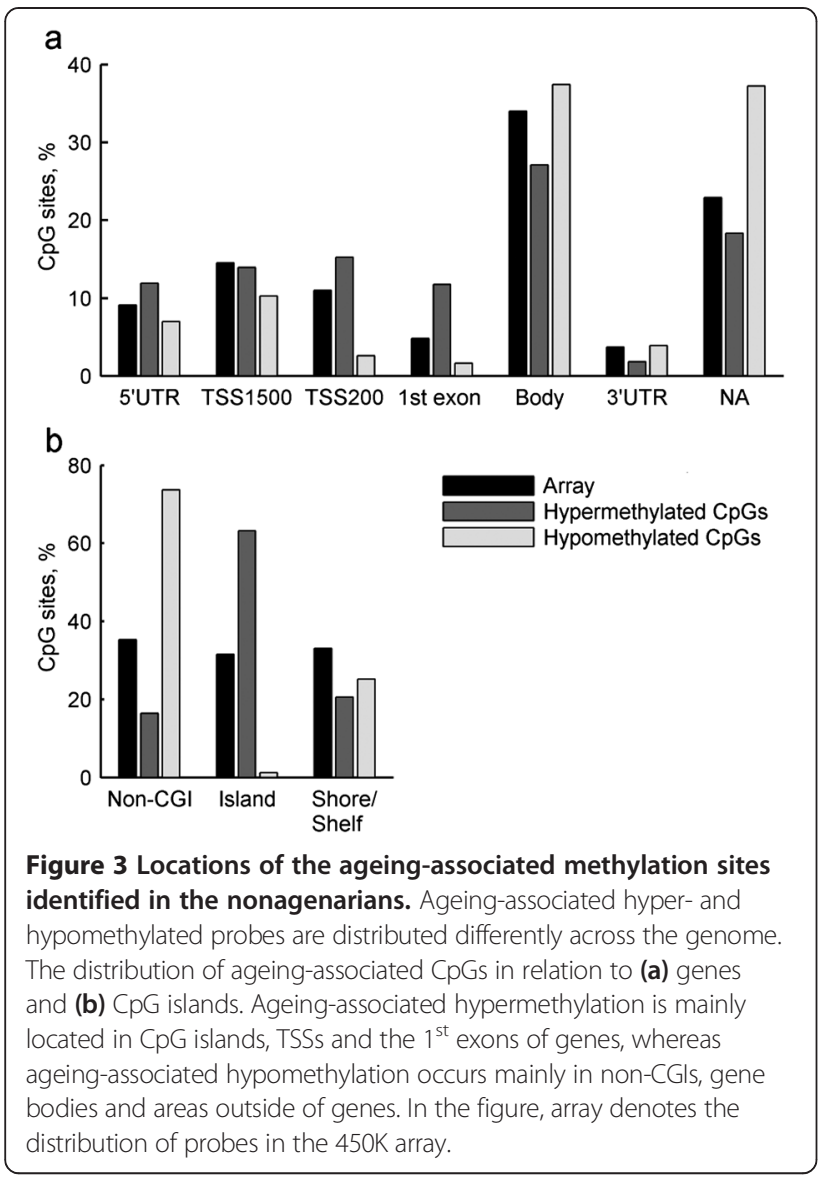

as covariates. The sex-associated sites that were also ageing-associated are listed in Additional file 9.

\section{Association between ageing-associated DNA methylation changes and gene expression}

We performed a correlation analysis between the level of methylation at ageing-associated CpGs and the expression level of genes in which these CpG sites were located. In nonagenarians, we identified 422 correlation pairs (Pearson correlation, Benjamini-Hochberg-corrected p-value $<0.05$ ) that consisted of 377 individual CpG sites and 233 individual genes (Additional file 10). The apparent discrepancy in these numbers is because a single $\mathrm{CpG}$ can be located in a region of overlapping transcripts, and there can be several CpGs within the coding region of a single transcript. In the young controls, we identified 50 expression-methylation correlation pairs (Pearson correlation, Benjamini-Hochberg-corrected p-value $<0.05$ ), consisting of 43 individual $\mathrm{CpGs}$ and 37 individual genes. In nonagenarians, 255 (60\%) of these correlated CpG-gene pairs showed an inverse correlation, and 167 (40\%) exhibited a direct correlation. In the young controls, these numbers were $46(92 \%)$ and $4(8 \%)$, respectively. Among the genes whose expression level was correlated with the level of DNA methylation, 23 were common to the nonagenarians and young controls, and in all cases, the direction of correlation was the same. We previously showed that 14 of the 233 genes identified in the present study were differentially expressed with age in both sexes and that an additional 14 were differentially expressed with age in either sex [35] (For details, see Additional file 10).

The correlated CpGs did not exhibit a similar distribution in the genome to the ageing-associated methylation sites. Those $\mathrm{CpG}$ sites whose methylation level correlated with the level of gene expression were concentrated in non-CGIs and on CpG island shores and shelves, whereas only a few (14.6\%) were located in CpG islands. In the non-CGIs, the majority of correlations were direct, whereas the opposite situation was observed in CpG islands and on island shores and shelves. With regard to regions within genes, the correlated $\mathrm{CpGs}$ were relatively evenly distributed. However, we identified an abundance of correlated CpGs within gene bodies (55\% of all correlated sites), where the majority of sites were directly correlated. In regions near a TSS (TSS200, from TSS

Table 3 Hypermethylation-specific GO function terms in nonagenarians

\begin{tabular}{lllll}
\hline GO term & Description & P-value & FDR q-value & Rank (out of 36) \\
\hline GO:0043565 & Sequence-specific DNA binding & $1.18 \mathrm{E}-32$ & $4.65 \mathrm{E}-29$ & 1 \\
GO:0001071 & Nucleic acid binding transcription factor activity & $9.38 \mathrm{E}-31$ & $1.85 \mathrm{E}-27$ & 2 \\
GO:0003700 & Sequence-specific DNA binding transcription factor activity & $2.22 \mathrm{E}-30$ & $2.92 \mathrm{E}-27$ & 3 \\
GO:0003677 & DNA binding & $6.48 \mathrm{E}-16$ & $6.38 \mathrm{E}-13$ & 4 \\
GO:0000981 & Sequence-specific DNA binding RNA polymerase II transcription factor activity & $2.6 \mathrm{E}-15$ & $2.05 \mathrm{E}-12$ & 5 \\
GO:0000976 & Transcription regulatory region sequence-specific DNA binding & $4.8 \mathrm{E}-13$ & $2.7 \mathrm{E}-10$ & 7 \\
GO:0044212 & Transcription regulatory region DNA binding & $7.92 \mathrm{E}-12$ & $3.47 \mathrm{E}-9$ & 9 \\
GO:0000975 & Regulatory region DNA binding & $2.22 \mathrm{E}-11$ & $8.75 \mathrm{E}-9$ & 10 \\
GO:0001067 & Regulatory region nucleic acid binding & $2.22 \mathrm{E}-11$ & $7.96 \mathrm{E}-9$ & 11
\end{tabular}

This table includes only the hypermethylation-specific GO function terms that form a common cluster, associated with DNA binding and transcription. The presented p-values are unadjusted and the threshold for significance is $1.27 \mathrm{e}-5$ (Bonferroni). The rank denotes the placement of a given GO term in the list of all significant GO terms. For all statistically significant GO function terms, see Additional file 3. 
Table 4 Hypermethylation-specific GO process terms in nonagenarians

\begin{tabular}{lllll}
\hline GO term & Description & P-value & FDR q-value & Rank (out of 265) \\
\hline GO:0048598 & Embryonic morphogenesis & $1.25 \mathrm{E}-22$ & $8.85 \mathrm{E}-20$ & 17 \\
GO:0048729 & Tissue morphogenesis & $2.99 \mathrm{E}-19$ & $1.64 \mathrm{E}-16$ & 22 \\
GO:0002009 & Morphogenesis of an epithelium & $6.94 \mathrm{E}-18$ & $3.22 \mathrm{E}-15$ & 26 \\
GO:0001763 & Morphogenesis of a branching structure & $1.84 \mathrm{E}-17$ & $7.65 \mathrm{E}-15$ & 29 \\
GO:0048754 & Branching morphogenesis of an epithelial tube & $1.26 \mathrm{E}-15$ & $3.09 \mathrm{E}-13$ & 49 \\
GO:0048562 & Embryonic organ morphogenesis & $6.12 \mathrm{E}-14$ & $1.1 \mathrm{E}-11$ & 67 \\
GO:0009887 & Organ morphogenesis & $1.13 \mathrm{E}-13$ & $1.92 \mathrm{E}-11$ & 71 \\
GO:0035107 & Appendage morphogenesis & $2.17 \mathrm{E}-12$ & $2.85 \mathrm{E}-10$ & 92 \\
GO:0035108 & Limb morphogenesis & $2.17 \mathrm{E}-12$ & $2.82 \mathrm{E}-10$ & 93 \\
GO:0030326 & Embryonic limb morphogenesis & $7.07 \mathrm{E}-12$ & $8.7 \mathrm{E}-10$ & 98 \\
GO:0035113 & Embryonic appendage morphogenesis & $7.07 \mathrm{E}-12$ & $8.61 \mathrm{E}-10$ & 99 \\
GO:0048704 & Embryonic skeletal system morphogenesis & $2.25 \mathrm{E}-11$ & $2.56 \mathrm{E}-9$ & 106 \\
GO:0048705 & Skeletal system morphogenesis & $1.04 \mathrm{E}-10$ & $1.11 \mathrm{E}-8$ & 113 \\
GO:0048732 & Gland development & $2.37 \mathrm{E}-10$ & $2.3 \mathrm{E}-8$ & 124
\end{tabular}

This table includes only the hypermethylation-specific GO process terms that form a common cluster, associated with development and morphogenesis. The presented p-values are unadjusted and the threshold for significance is 4.15e-6 (Bonferroni). The rank denotes the placement of a given GO term in the list of all significant GO terms. For all statistically significant GO process terms, see Additional file 5.

to -200 nucleotides upstream of TSS), directly correlated CpGs were almost completely absent (Additional file 11).

To analyse the processes associated with the genes that displayed a correlation between expression and methylation levels, we performed GO term analysis and IPA for the nonagenarians. We identified $20 \mathrm{GO}$ process terms (Bonferronicorrected p-value $<0.05)$, of which $6(30 \%)$ were immune system associated. Numerous immune system pathways were also identified when considering GO process terms that were more loosely associated with these genes (Benjamini-Hochberg-corrected p-value <0.05), where 39 of 121 (32\%) statistically significant GO process terms were immune system associated (Additional file 12). Only one GO function term (GO:0005515 Protein binding) was associated with the correlated CpGs. In addition to the immune system, pathways related to the reaction to the environment were affected. Ingenuity canonical pathway analysis revealed 15 canonical pathways (Benjamini-Hochberg-corrected p-value <0.05) (Table 6), the majority of which were directly immune system associated (Crosstalk between Dendritic Cells and Natural Killer Cells, Antigen Presentation Pathway, Fcy Receptormediated Phagocytosis in Macrophages and Monocytes, $T$ Helper Cell Differentiation) or associated with cytoskeleton remodelling and endocytosis (Integrin Signalling, Actin Cytoskeleton Signalling, Tec Kinase Signalling, Paxillin Signalling, Caveolar-mediated Endocytosis Signalling).

\section{Discussion}

Ageing-associated DNA methylation changes; single CpG sites and their location and function

Here, we present the results of our ageing-associated DNA methylation analysis. In summary, our results were obtained with a 450K array using PBMCs collected from nonagenarians and young controls. The study subjects were analysed as two age groups, and we used two different statistical methods to verify that the ageing-associated methylation sites identified had a prominent difference in the level of methylation between the age groups and that this difference was not due to changes in leukocyte proportions. The proportions of leukocytes were measured via FACS. We also added a layer of information by including gene expression data from the same individuals. The small number of young controls is a potential limitation in our study; thus, the results should be interpreted accordingly.

In previous ageing-methylation studies, the age range of the oldest study subjects has typically been from 70 to 80 years of age $[17,19-22,25,26]$, and the youngest age group to be included has ranged from new-borns $[22,28]$ to 50 years of age $[20,25]$. In studies in which subjects over 90 years old have been analysed, these individuals represented a minority of the study population or the overall sample size has been small $[18,27,28,36]$. Hence, a strength of our study is the large number of the oldest-old individuals homogenous in terms of age. In addition, our study population represents the two extremities of adulthood, and as age was used as a dichotomous variable we were able to identify both changes occurring linearly with age as well as changes that occur in either end of the spectrum. The DNA methylation studies performed with 27K arrays [17-22] fail to capture methylation changes outside gene promoters, yet our results, as well as those of others $[25,28,36]$, show that ageing-associated changes are not restricted to gene promoters. In contrast to our study, previous reports combining methylation and expression 
Table 5 Hypermethylation-specific GO process terms in nonagenarians

\begin{tabular}{|c|c|c|c|c|}
\hline GO term & Description & P-value & FDR q-value & Rank (out of 265) \\
\hline GO:0045935 & Positive regulation of nucleobase-containing compound metabolic process & $7.07 \mathrm{E}-17$ & $2.37 \mathrm{E}-14$ & 36 \\
\hline GO:0051173 & Positive regulation of nitrogen compound metabolic process & $1.06 \mathrm{E}-16$ & $3.44 \mathrm{E}-14$ & 37 \\
\hline GO:0031328 & Positive regulation of cellular biosynthetic process & $2.74 \mathrm{E}-16$ & 8.47E-14 & 39 \\
\hline GO:0009891 & Positive regulation of biosynthetic process & $3.61 \mathrm{E}-16$ & $1.06 \mathrm{E}-13$ & 41 \\
\hline GO:0045893 & Positive regulation of transcription, DNA-templated & $5.05 \mathrm{E}-16$ & $1.35 \mathrm{E}-13$ & 45 \\
\hline GO:0019219 & Regulation of nucleobase-containing compound metabolic process & 7.72E-16 & $2.02 \mathrm{E}-13$ & 46 \\
\hline GO:0010628 & Positive regulation of gene expression & $1.11 \mathrm{E}-15$ & $2.78 \mathrm{E}-13$ & 48 \\
\hline GO:0006357 & Regulation of transcription from RNA polymerase II promoter & $5.05 \mathrm{E}-15$ & 1.15E-12 & 53 \\
\hline GO:0031326 & Regulation of cellular biosynthetic process & $1.07 \mathrm{E}-14$ & 2.27E-12 & 57 \\
\hline GO:0051171 & Regulation of nitrogen compound metabolic process & $1.28 \mathrm{E}-14$ & $2.66 \mathrm{E}-12$ & 58 \\
\hline GO:0009889 & Regulation of biosynthetic process & $1.45 \mathrm{E}-14$ & $2.96 \mathrm{E}-12$ & 59 \\
\hline GO:0051254 & Positive regulation of RNA metabolic process & $1.8 \mathrm{E}-14$ & $3.56 \mathrm{E}-12$ & 61 \\
\hline GO:0006355 & Regulation of transcription, DNA-templated & $1.98 \mathrm{E}-14$ & $3.85 \mathrm{E}-12$ & 62 \\
\hline GO:1902680 & Positive regulation of RNA biosynthetic process & $2.06 \mathrm{E}-14$ & $3.93 \mathrm{E}-12$ & 63 \\
\hline GO:0045944 & Positive regulation of transcription from RNA polymerase II promoter & $2.94 \mathrm{E}-14$ & $5.45 \mathrm{E}-12$ & 65 \\
\hline GO:0010557 & Positive regulation of macromolecule biosynthetic process & $6.72 \mathrm{E}-14$ & $1.19 \mathrm{E}-11$ & 68 \\
\hline GO:0031323 & Regulation of cellular metabolic process & $1.09 \mathrm{E}-13$ & $1.87 \mathrm{E}-11$ & 70 \\
\hline GO:2001141 & Regulation of RNA biosynthetic process & $1.37 \mathrm{E}-13$ & $2.29 \mathrm{E}-11$ & 72 \\
\hline GO:0031325 & Positive regulation of cellular metabolic process & $2.22 \mathrm{E}-13$ & 3.57E-11 & 75 \\
\hline GO:0045934 & Negative regulation of nucleobase-containing compound metabolic process & $2.8 \mathrm{E}-13$ & 4.39E-11 & 77 \\
\hline GO:0031327 & Negative regulation of cellular biosynthetic process & $3.75 \mathrm{E}-13$ & $5.8 \mathrm{E}-11$ & 78 \\
\hline GO:0009893 & Positive regulation of metabolic process & $3.84 \mathrm{E}-13$ & $5.85 \mathrm{E}-11$ & 79 \\
\hline GO:0009890 & Negative regulation of biosynthetic process & $3.84 \mathrm{E}-13$ & $5.78 \mathrm{E}-11$ & 80 \\
\hline GO:0000122 & Negative regulation of transcription from RNA polymerase II promoter & $5.25 \mathrm{E}-13$ & $7.72 \mathrm{E}-11$ & 82 \\
\hline GO:0051252 & Regulation of RNA metabolic process & $9.69 \mathrm{E}-13$ & $1.39 \mathrm{E}-10$ & 84 \\
\hline GO:0051172 & Negative regulation of nitrogen compound metabolic process & $1.07 \mathrm{E}-12$ & $1.52 \mathrm{E}-10$ & 85 \\
\hline GO:2000112 & Regulation of cellular macromolecule biosynthetic process & $1.14 \mathrm{E}-12$ & $1.6 \mathrm{E}-10$ & 86 \\
\hline GO:0080090 & Regulation of primary metabolic process & $1.39 \mathrm{E}-12$ & $1.92 \mathrm{E}-10$ & 87 \\
\hline GO:0010629 & Negative regulation of gene expression & $2.02 \mathrm{E}-12$ & $2.68 \mathrm{E}-10$ & 91 \\
\hline GO:0010556 & Regulation of macromolecule biosynthetic process & $3.09 \mathrm{E}-12$ & $3.96 \mathrm{E}-10$ & 94 \\
\hline GO:0045892 & Negative regulation of transcription, DNA-templated & 4.17E-12 & $5.29 \mathrm{E}-10$ & 95 \\
\hline GO:1902679 & Negative regulation of RNA biosynthetic process & $4.83 \mathrm{E}-12$ & $6.07 \mathrm{E}-10$ & 96 \\
\hline GO:0019222 & Regulation of metabolic process & $1.56 \mathrm{E}-11$ & 1.84E-9 & 102 \\
\hline GO:0051253 & Negative regulation of RNA metabolic process & $1.94 \mathrm{E}-11$ & $2.25 \mathrm{E}-9$ & 104 \\
\hline GO:0010468 & Regulation of gene expression & $1.35 \mathrm{E}-10$ & $1.39 \mathrm{E}-8$ & 117 \\
\hline GO:0010558 & Negative regulation of macromolecule biosynthetic process & 1.39E-10 & $1.41 \mathrm{E}-8$ & 119 \\
\hline GO:0010604 & Positive regulation of macromolecule metabolic process & 1.49E-10 & $1.46 \mathrm{E}-8$ & 123 \\
\hline GO:2000113 & Negative regulation of cellular macromolecule biosynthetic process & $3.62 \mathrm{E}-10$ & $3.38 \mathrm{E}-8$ & 129 \\
\hline
\end{tabular}

This table includes only the hypermethylation-specific GO process terms that form a common cluster, associated with nucleotide metabolism, RNA metabolism and transcription. The presented p-values are unadjusted and the threshold for significance is 4.15e-6 (Bonferroni). The rank denotes the placement of a given $\mathrm{GO}$ term in the list of all significant GO terms. For all statistically significant $\mathrm{GO}$ process terms, see Additional file 5.

data have relied on individuals from different study cohorts $[27,36]$. A group of studies have also tried to identify a small set of methylation sites that could be used to construct an ageing signature [22,23,25,27]. However, by focusing on a broader set of ageing-associated methylation sites, the mechanisms of ageing can be more thoroughly examined. Given that published ageing-methylation studies have been conducted using various age ranges and 


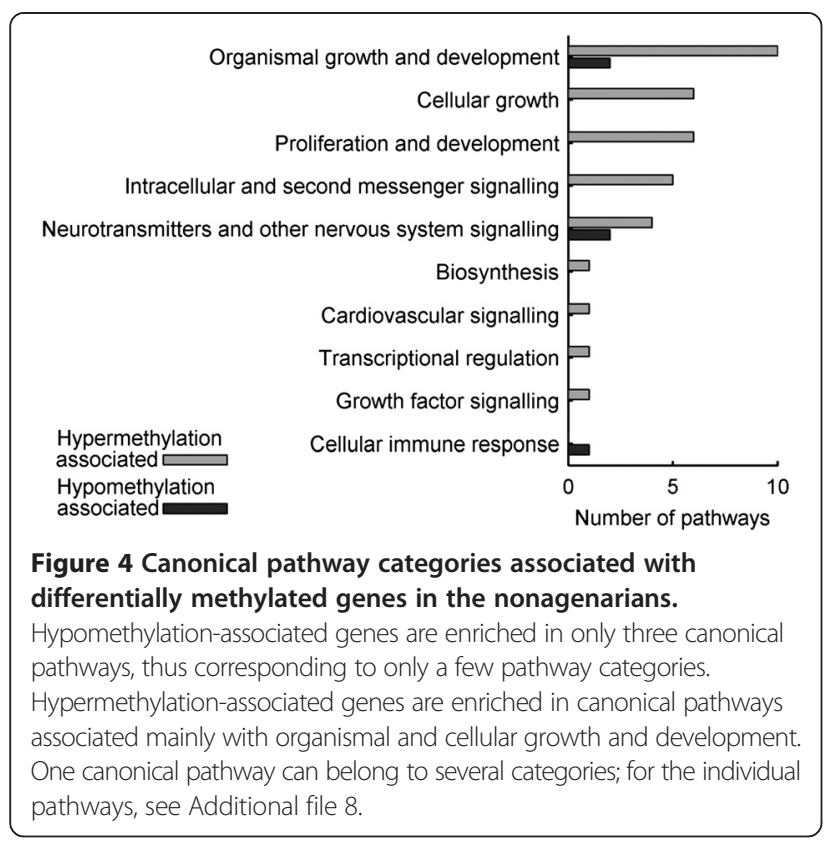

statistical methods, discrepancies in the results are most likely due to both biological and statistical factors.

The main characteristics of the ageing-associated methylation sites identified in the present study are presented in Table 7. We identified 8540 high-confidence CpG sites that show a large difference in methylation levels between nonagenarians and young controls and that present high statistical significance in a regression model adjusted for the leukocyte proportion. A slight majority (54\%) of the identified sites were hypomethylated in the nonagenarians. Among the top-ranking ageing-associated methylation changes that have been reported with a high frequency, ELOVL2 (cg16867657, cg24724428), PENK (cg04598121), FHL2 (cg22454769, cg24079702, cg06639320) EDARADD (cg09809672), KLF14 (cg04528819, cg07955995) and OTUD7A (cg04875128) were also identified in our study. Of these genes, only EDARADD was hypomethylated in the nonagenarians compared with the controls. As reported by Steegenga et al. [24], among 8 previous studies analysing the association of ageing and DNA methylation changes in PBMCs [17,19-21,25-28], only 529 probes were reported to be affected by age by more than one research group. Of these probes, our analysis identified 105. Interestingly, the majority of frequently reported CpG sites are hypermethylated with increasing age. Among the $151 \mathrm{CpG}$ sites (148 of which are present in $450 \mathrm{~K}$ ) reported to be associated with ageing by more than 3 groups [24], 77\% were hypermethylated. Of the $105 \mathrm{CpG}$ sites that are frequently reported and were identified in our study, 79\% (83/105) were hypermethylated.

The functional roles of the 10 most frequently reported ageing-associated methylation sites are currently unclear, as they are not associated with a common, ageing-related process. According to our results, the genes associated with these sites are not enriched under a common GO term or

Table 6 Canonical pathways associated with genes whose expression levels correlate with the level of DNA methylation in nonagenarians

\begin{tabular}{|c|c|c|c|}
\hline Ingenuity canonical pathways & p-value (B-H corrected) & Ratio & Molecules \\
\hline Integrin signalling & 0.016 & 0.054 & ITGB1,PTK2,RAP2A,FYN,PAK1,RALA,ACTA2,ITGA6,CAPN2,ITGAL,ACTN1 \\
\hline Actin cytoskeleton signalling & 0.017 & 0.048 & ITGB1,PTK2,TIAM1,PAK1,F2R,ACTA2,TRIO,PDGFD,GSN,ARHGAP24,ACTN1 \\
\hline Tec kinase signalling & 0.019 & 0.051 & STAT4,ITGB1,PTK2,FYN,GNAI3,GNB4,PAK1,ACTA2,HCK \\
\hline $\begin{array}{l}\text { Agrin interactions at } \\
\text { neuromuscular junction }\end{array}$ & 0.019 & 0.090 & ITGB1,PTK2,PAK1,ACTA2,ITGA6,ITGAL \\
\hline Paxillin signalling & 0.020 & 0.064 & ITGB1,PTK2,PAK1,ACTA2,ITGA6,ITGAL,ACTN1 \\
\hline Reelin signalling in neurons & 0.026 & 0.073 & ITGB1,FYN,HCK,ITGA6,ARHGEF11,ITGAL \\
\hline Phospholipase C signalling & 0.030 & 0.041 & ITGB1,FYN,GNB4,RALA,AHNAK,SYK,MEF2C,ARHGEF11,PLD6,CREB5 \\
\hline Germ cell-sertoli cell junction signalling & 0.030 & 0.051 & ITGB1,PTK2,TGFBR2,PAK1,ACTA2,ITGA6,GSN,ACTN1 \\
\hline $\begin{array}{l}\text { Crosstalk between dendritic cells and } \\
\text { natural killer cells }\end{array}$ & 0.030 & 0.066 & IFNG,ACTA2,CD86,HLA-F,ITGAL,CCR7 \\
\hline Protein kinase A signalling & 0.030 & 0.035 & $\begin{array}{l}\text { TGFBR2,PTK2,GNB4,GNAI3,TCF4,PTPN7,YWHAG,DUSP10, } \\
\text { RYR1,LEF1,CREB5,PTPRM,SIRPA }\end{array}$ \\
\hline Antigen presentation pathway & 0.030 & 0.100 & PSMB9,IFNG,HLA-F,HLA-DPB1 \\
\hline $\begin{array}{l}\text { Fcy receptor-mediated phagocytosis in } \\
\text { macrophages and monocytes }\end{array}$ & 0.030 & 0.063 & FYN,PAK1,SYK,ACTA2,HCK,PLD6 \\
\hline T helper cell differentiation & 0.037 & 0.073 & STAT4,TGFBR2,IFNG,IFNGR2,CD86 \\
\hline Ephrin receptor signalling & 0.038 & 0.041 & ITGB1,PTK2,FYN,GNAI3,GNB4,PAK1,PDGFD,CREB5 \\
\hline Caveolar-mediated endocytosis signalling & 0.044 & 0.062 & ITGB1,FYN,ACTA2,ITGA6,ITGAL \\
\hline
\end{tabular}


Table 7 Characteristics of ageing-associated methylation sites

\begin{tabular}{lll}
\hline & Hypermethylated & Hypomethylated \\
\hline $\mathrm{n}$ & 3925 & 4615 \\
CpG island location & CpG islands & Non-CGl \\
Genomic location & TSS, 1st exon & $\begin{array}{l}\text { Gene body, } \\
\text { outside genes }\end{array}$ \\
Associated genes & 1832 & 2057 \\
GO function terms & 36 & 27 \\
GO process terms & 265 & 53 \\
Canonical pathways (IPA) & 19 & 3 \\
Transcription factors & 24 & 1 \\
\hline
\end{tabular}

The CpG island location and genomic location refer to the sites where hyper- and hypomethylated sites are most abundant. Notably, there are more hypomethylated CpG sites compared with hypermethylated CpG sites and therefore also more hypomethylation-associated genes, yet the hypermethylation-associated genes are enriched in more GO terms and canonical pathways, and they share more common transcription factors.

in common canonical pathways. Only FHL2, PENK and OTUD7A are included in any identified GO term, and none of them are included in affected canonical pathways. The methylation levels of frequently reported CpGs are not correlated with the expression levels of the corresponding genes. For $E D A R A D D$, we identified an additional CpG site (cg18964582) that was differentially methylated between nonagenarians and young controls, located within TSS1500, where there is an inverse correlation between the methylation level and the expression level. However, based on previous findings and our results, it appears that the frequently reported ageing-associated $\mathrm{CpG}$ sites are not strongly associated with known ageing-related mechanisms but could instead represent a cellular chronological clock mechanism.

Our results revealed an enrichment of ageing-associated hypermethylation at $\mathrm{CpG}$ islands, whereas hypomethylation was enriched in non-CGIs and was almost totally absent from CpG islands. These findings are in line with previously reported results $[16,24,25,28,36,37]$. The majority of CpG sites are not initially methylated in CpG islands, and the change observed during ageing is hypermethylation. The opposite is true for regions with few CpG sites that initially are heavily methylated, and the non-CGIs are associated with hypomethylation. These results support the notion that the normal maintenance of DNA methylation patterns is disrupted with ageing [38]. As both hypomethylation and hypermethylation occur with ageing, it appears that both de novo methylation processes, mediated by DNMT3A and DNMT3B methyltransferases, and the maintenance of existing DNA methylation, mediated by $D N M T 1$, are disrupted with ageing. Interestingly, our results identified 4 CpG sites in DNMT3A that were ageing associated (cg00050692, which was hypomethylated, and cg15302376, cg15843262 and cg26544247, which were hypermethylated in the nonagenarians). However, there was no correlation between the level of methylation and DNMT3A expression.

Our results showed that not only are ageing-associated hyper- and hypomethylation found at different genomic sites but that these changes are also found in genes associated with different functions. Our findings further revealed that ageing-associated hypermethylation is concentrated in genes associated with developmental processes as well as DNA-binding and transcription of genes, whereas hypomethylation is not enriched among a specific set of genes. Johansson et al. [36], Rakyan et al. [19] and Florath et al. [25] previously reported the association of hypermethylation with developmental processes and DNA binding. As DNA methylation regulates DNA transcription, it is interesting that the genes required during this process are differentially methylated with ageing. In comparison with ageing-associated hypomethylation, hypermethylation appears to be a more regulated process, as no strongly hypomethylation-specific functions or processes were identified in this study.

It is notable that while the individual sites reported to be ageing associated differ to some extent between studies, the results regarding their locations in the genome and the molecular functions with which they are associated are more uniform. Single highly significant $\mathrm{CpG}$ sites have also been reported in various studies, including sites located in the ELOVL2 and FLH2 genes. Common ageing-associated DNA methylation changes can also be observed across different tissues [6,23]. Thus, it appears that at least some fraction of ageing-associated DNA methylation changes is caused by programmed or pseudoprogrammed changes that occur in a similar manner across tissues and individuals. As certain processes and sites are reported frequently, it can be hypothesised that these sites and processes represent clock-like changes associated with ageing. For example, a strong association with chronological age has been shown for ELOVL2 (cg16867657) [25,26,36]. However, it remains to be investigated whether these sites are only associated with chronological age or if there are also associations with phenotypic changes related to (successful) ageing. If these frequently reported sites are only markers of chronological age, markers of biological age are yet to be identified.

\section{The role of cell proportions in DNA methylation studies}

The majority of DNA methylation and expression studies are performed with whole blood or PBMCs due to the accessibility of these tissue types. However, PBMCs consist of various cell types, and different individuals can exhibit differences in the proportions of different cell populations. Ageing is known to be associated with changes in the proportions of $\mathrm{T}$ cells [2,39]. Furthermore, the different leukocyte subtypes show differences in their DNA methylation levels [40], and changes in 
DNA methylation are known to be one of the factors regulating lineage development in leukocytes [41].

Previous reports have claimed that differences in the proportions of leukocytes do not cause bias in methylation analyses $[17,21,22]$. However, contradictory reports have also been published [42], and recently it has been systematically shown that differences in leukocyte proportions should be taken into consideration when analysing ageingassociated methylation differences [29]. Our PCA revealed that the largest percentage of the variation in our methylation data was associated with the proportions of different leukocyte subtypes (Figure 1).

\section{The role of sex in ageing-associated DNA methylation studies}

According to our results, sex does not have a large effect on ageing-associated DNA methylation changes in autosomes, as we identified only 7 CpG sites for which sex, in addition to age group, was a significant covariate in the regression model. However, the small number of male samples in our control population may have precluded the identification of ageing-associated sex differences. Nevertheless, Johansson et al. [36] and McClay et al. [37] previously reported similar findings in studies focusing on individual sites associated with ageing. In studies where methylation profiles have been used to predict age, however, the methylome has been shown to age more rapidly in men than in women [22,27]. DNA methylation is believed to mediate the long-term regulation of gene expression [13], and it is therefore interesting to note that sex differences appear to be mediated via mechanisms other than DNA methylation. Apparently, the effects of sex observed in methylome studies predicting age are small global effects rather than large changes at a limited number of sites. We have previously reported [35] that there are sex-specific differences in the gene expression changes associated with ageing, but based on the results of the present study, these expression differences are not regulated by DNA methylation.

\section{The role of zinc-associated proteins in ageing}

We observed a clear enrichment of hypermethylation on chromosome 19, which seems to be due to the abundance of zinc finger proteins on this chromosome. The increased methylation of zinc finger genes on chromosome 19 has previously been observed in oropharyngeal squamous cell carcinoma [43], and similarities between the methylation changes that occur in ageing and cancer have been demonstrated in multiple studies $[20,21,23]$. It has recently been proposed that the zinc finger proteins on chromosome 19 have specifically evolved to repress endogenous retroviruses (ERVs) [44]. On the other hand, the expression of ERVs has been associated with ageing in mice $[45,46]$. Hence, the hypermethylation of zinc finger genes observed with ageing offers an explanation for why ERVs are able to be expressed with advancing age. One of the zinc finger genes predicted to repress ERVs by Lukic et al. [44] was ZNF154. We identified 10 CpGs within this gene as being hypermethylated in the nonagenarians, and we identified a strong negative correlation between the level of methylation and the expression of this gene, indicating that its expression is truly downregulated in the aged individuals. Both ageing and cancer are associated with genomic instability [1], and the role of active ERVs in inducing this genomic instability with increasing age could be analogous to that proposed in cancer [47].

Zinc-coordinating transcription factors were also enriched among the TFs predicted to regulate hypermethylationassociated genes in this study, as 12 out of the 24 identified TFs were zinc coordinating. Zinc has been associated with various processes that are known to be regulated during ageing, such as immune function, DNA repair mechanisms, cell proliferation, apoptosis and transcription $[48,49]$.

\section{The association between ageing-associated DNA methylation changes and gene expression}

We sought to examine the relationship between ageingassociated DNA methylation changes and gene expression levels. Compared with previous studies, a key asset of our study is that methylation and gene expression data were available from the same samples. Those ageing-associated methylation sites in which the level of methylation is associated with the level of gene expression are concentrated in non-CGIs and on shores and shelves, as well as in gene body regions. Similar findings have been reported by Zilbauer et al. [40]. Gene-body methylation has been proposed to affect gene expression via splicing and alternative start site usage $[13,50]$. It is important to note that many previous studies examining DNA methylation changes during ageing have been performed using the Illumina $27 \mathrm{~K}$ array, where the majority of the probes are located in promoter regions. In these studies, the effects of gene-body methylation on gene expression levels remained unidentified.

The identified genes that display expression-methylation correlations are strongly enriched in immunological processes and in cytoskeletal remodelling and endocytosis. Cytoskeletal remodelling is required for leukocyte activation, migration and phagocytosis [51]. The results imply that some fraction of ageing-associated immune system changes may be regulated by DNA methylation. Defects in the immune system are a hallmark of ageing, leading to increased susceptibility to infectious diseases, cancer and ultimately death [1]. DNA methylation typically regulates long-term trends in gene expression $[11,13]$, and the possibility that immune system-related processes may be locked in a particular state by DNA methylation could 
offer one explanation as to why the immune system of elderly individuals is not able to respond appropriately to various insults.

We found that only a minority of ageing-associated CpG sites showed an association between methylation and expression levels. Furthermore, only a minority of these genes have been identified as differentially expressed between nonagenarians and young individuals [35]. Previous studies have also found a limited number of associations between ageing-associated DNA methylation changes and gene expression levels $[21,23,27,36,42,52]$. Due to the methods applied in the present study, not all the effects of DNA methylation on gene expression could be detected; this limitation is also true for previously reported results. The textbook case of DNA methylation regulating gene expression (the methylation of a promoter and silencing of a gene) remains undetected in many cases because in an array analysis, an unexpressed gene shows no signal that can be distinguished from background and is therefore typically omitted from the analysis. Additionally, in the present study, the methylation level of each CpG was correlated separately with gene expression. In CpG island regions in particular, the effect of DNA methylation changes on gene expression could be observed when a cluster of closely located CpG sites were analysed as a whole. The effects of CpG sites that are not located in the regulated gene itself also remain unidentified. The short list of methylation-gene expression associations linked to ageing reported herein and previously by others should be interpreted as a defined set of one type of methylationgene expression associations, and it should be assumed that other types of mechanisms exist and require different methodologies to be identified.

\section{Conclusions}

Based on the results presented here, it appears that ageingassociated hyper- and hypomethylation are distinct processes, both in terms of their causes and consequences. We suggest that hypermethylation is an active process, caused by programmed or pseudo-programmed ageing processes, and that hypermethylation is strongly associated with chronological age. Ageing-associated hypomethylation, however, is a passive process caused by stochastic or environmental effects and is associated with biological age, i.e., the phenotype of the individual. Whether the underlying cause of ageing is programmed, pseudo-programmed or due to the accumulation of molecular damage has been widely discussed in the literature. Given that evidence supporting each theory can be found, it is plausible that these mechanisms all contribute to the ageing process but possibly affect different aspects [3-5].

First, hypermethylation is an active process that consumes energy as new methyl groups are added to DNA by DNA methyltransferases. Hypomethylation can also be an active process in some cases, but contrary to hypermethylation, it may occur passively as well $[53,54]$. The most frequently reported ageing-associated DNA methylation changes (for example in ELOVL2) that are repeated across tissues and study populations, thus implying programmed changes, are hypermethylation events. In studies where chronological age has been explained in association with DNA methylation levels, it has been found that at sites showing the strongest correlation with chronological age, methylation increases with age $[25,26]$. The ageingassociated hypermethylated sites form common groups with regard to cellular processes and functions. According to the results of the present study, hypermethylationassociated genes are predicted to be regulated by a common group of transcription factors and are also enriched in common GO terms, whereas hypomethylationassociated genes do not to appear to form common groups. The top-ranking ageing-associated sites are hypermethylated, but hypomethylated sites are more numerous. This difference becomes more significant when the threshold of significance is lowered; of the 8540 sites identified here, $54 \%$ were hypomethylated, but among the 45507 sites identified with the regression model, $64 \%$ were hypomethylated. Johansson et al. [36] also reported an excess of hypomethylation over hypermethylation with ageing.

Global hypomethylation has been associated with an increasing risk of frailty [55], but very few other associations between phenotype and DNA methylation have been reported [17]. However, this may be due to technical concerns, as the study by Bell et al. [17] was performed with the $27 \mathrm{~K}$ array, which almost exclusively contains promoter-associated probes that are not methylated at baseline and can therefore primarily acquire hypermethylation. Phenotype association studies performed with the $450 \mathrm{~K}$ array or using sequencing techniques are necessary to clarify if hypomethylation is associated with typical ageing-associated phenotypes.

The role of DNA methylation is known to differ depending on its location in the genome. Thus, it would not be surprising if different DNA methylation changes in the genome are affected by different ageing mechanisms. As DNA methylation analyses are complicated by the different effects of methylation sites at different genomic positions and by the cumulative effects of nearby CpG sites, all possible known biases, such as the proportions of leukocytes, should be accounted for in DNA methylation analyses.

\section{Methods}

\section{Study population}

The study population consisted of 146 nonagenarians (females $\mathrm{n}=103$, males $\mathrm{n}=43$ ) participating in the Vitality $90+$ study and 30 young, healthy controls (aged 19-30 years, median 22.5 years; females $n=21$, males 
$n=9)$. Gene expression data were available for all the individuals, and methylation data were available for 122 nonagenarians ( $\mathrm{n}=89$ females and $\mathrm{n}=33$ males) and 21 young controls ( $\mathrm{n}=14$ females and $\mathrm{n}=7$ males), and data on cell proportions were available for 115 nonagenarians ( $\mathrm{n}=84$ females and $\mathrm{n}=31$ males) and all 30 of the young controls. All the study subjects were of Western European descent. The Vitality $90+$ study is an ongoing prospective population-based study that includes both home-dwelling and institutionalised individuals aged 90 years or more who live in the city of Tampere, Finland. The recruitment and characterisation of the participants were performed as previously reported for earlier Vitality 90+ study cohorts [56]. In this study, we included only individuals born in 1920, and the evaluated samples were collected in the year 2010. The nonagenarians included in the study had not had any infections or received any vaccinations in the 30 days prior to blood sample collection. The young controls consisted of healthy laboratory personnel who did not have any medically diagnosed chronic illnesses, were non-smokers and had not had any infections or received any vaccinations within the two weeks prior to blood sample collection. The study participants provided their written informed consent. The study has been conducted according to the principles expressed in the declaration of Helsinki, and the study protocol was approved by the ethics committee of the city of Tampere (1592/403/1996).

\section{Sample collection}

The blood samples were collected into EDTA-containing tubes by a trained medical student during a home visit. All the blood samples were drawn between 8 am and 12 am. The samples were directly subjected to leucocyte separation on a Ficoll-Paque density gradient (Ficoll-Paque ${ }^{\mathrm{Tx}}$ Premium, cat. no. 17-5442-03, GE Healthcare Bio-Sciences AB, Uppsala, Sweden). The PBMC layer was collected, and a subset of the cells was suspended in $150 \mu \mathrm{l}$ of RNAlater solution (Ambion Inc., Austin, TX, USA) for use in a gene expression microarray analysis. Cells that were to be subjected to FACS analysis and DNA extraction were suspended in $1 \mathrm{ml}$ of a freezing solution (5/8 FBS, 2/8 RPMI-160 medium, 1/8 DMSO) (FBS cat. no. F7524, Sigma-Aldrich, MO, USA; RPMI: cat. no. R0883, Sigma-Aldrich, MO, USA; DMSO: cat. no. 1.02931.0500, VWR, Espoo, Finland).

\section{DNA extraction}

DNA was extracted from PBMCs using the QIAamp DNA Mini kit (Qiagen, CA, USA), following the manufacturer's instructions for the spin protocol. The DNA was eluted in $60 \mu \mathrm{l}$ of AE elution buffer and stored at $-20^{\circ} \mathrm{C}$. The concentration and quality of the DNA was assessed with the Qubit dsDNA HS Assay (Invitrogen, Eugene, OR, USA).

\section{RNA extraction}

For RNA extraction, equal amounts of PBS and RNAlater were added to the cell suspension and then removed via centrifugation, leaving only the cell pellet. RNA was purified using an miRNeasy mini kit (Qiagen, CA, USA), according to the manufacturer's protocol, with on-column DNase digestion (AppliChem $\mathrm{GmbH}$, Darmstadt, Germany). The concentration and quality of the RNA were assessed with the Agilent RNA 6000 Nano Kit on an Agilent 2100 Bioanalyzer (Agilent Technologies, CA, USA).

\section{FACS}

The proportions of different lymphocyte populations were determined through FACS analysis (BD FACSCanto II), and the results were analysed with BD FACS Diva, version 6.1.3 (BD Biosciences, Franklin Lakes, NJ, USA). The antibodies employed in this analysis were FITC-CD14 (cat. no. 11-0149), PerCP-Cy5.5-CD3 (45-0037), APCCD28 (17-0289) (eBioscience, San Diego, CA, USA), PE-Cy ${ }^{\text {tm }} 7-C D 4$ (cat. no. 557852) and APC-Cy ${ }^{\text {tm}} 7-C D 8$ (557834) (BD Biosciences).

\section{Expression array}

Labelled cRNA was prepared from $330 \mathrm{ng}$ of total RNA using the Illumina TotalPrep RNA Amplification Kit (Ambion Inc., TX, USA) with overnight incubation according to the manufacturer's protocol. The quality of the labelled cRNA was determined using a 2100 Bioanalyzer (Agilent Technologies). In total, $1500 \mathrm{ng}$ of labelled cRNA was hybridised overnight to a HumanHT-12 v4 Expression BeadChip (Cat no. BD-103-0204, Illumina Inc., CA, USA), according to the Illumina protocol, in the Core Facility of the Department of Biotechnology of the University of Tartu. Samples were assigned to the arrays in a randomised order. The chips were scanned using Beadscan (Illumina Inc.).

\section{Methylation array}

Genome-wide DNA methylation profiling was performed at the Institute for Molecular Medicine Finland (FIMM) Technology Centre of the University of Helsinki in two batches (time interval, 6 months). Bisulfite conversion of $1 \mu \mathrm{g}$ of DNA was performed using the EZ-96 DNA Methylation Kit (Zymo Research, Irvine, CA, USA) according to manufacturer's instructions. A 4- $\mu$ l aliquot of bisulphite-converted DNA was subjected to whole-genome amplification and then enzymatically fragmented and hybridised to the Infinium HumanMethylation450 BeadChip (Illumina, San Diego, CA, USA) according to manufacturer's protocol. Samples were assigned to the arrays in a randomised order. The BeadChips were scanned with the iScan reader (Illumina). 


\section{Preprocessing of the methylation microarray data}

The methylation data were preprocessed as a methylumiset object using $\mathrm{R}$ software with the wateRmelon arrayspecific package from Bioconductor [57]. The annotation information was based on the GRCh37/hg19 genome assembly from February 2009. Prior to any processing, all unspecific or polymorphic sites $(n=76775)$ were removed based on database information [58]. Samples and target sites of a technically poor quality were filtered out by excluding sites with a beadcount of $<3$ in $5 \%$ of the samples $(\mathrm{n}=526)$ and sites for which $1 \%$ of the samples showed a detection p-value $>0.05(n=740)$. Background correction and quantile normalisation via the dasen method were conducted individually for the two applied chemistries (Infinium I and II) as well as for the intensities of methylation $(\mathrm{m})$ and un-methylation $(\mathrm{u})$. After dasen treatment, the $\mathrm{u}$ and $\mathrm{m}$ intensities were transformed to beta $(\beta)$ and $M$ values. $\beta$ is the ratio of the methylated probe $(m)$ intensities to the overall intensities $(m+u+\alpha)$, where $\alpha$ is the constant offset, 100 . Thus, $\beta$ ranges linearly from 0 (nonmethylated, $0 \%$ ) to 1 (completely methylated, 100\%). The $\beta$ values were further transformed into $M$ values using the equation $\log 2(\beta /(1-\beta))$. Next, the batch effect of the chemistries was adjusted using the BMIQ method, which is based on beta mixture models and the EM algorithm [59]. Several visualisation styles were used to verify the quality of the preprocessed data, such as boxplots from the raw intensities, Kernel density plots in the chemistry correction procedure and PCA plots (see Additional file 13). The batch effect of two laboratory days (time interval of 6 months) was confirmed via PCA (PC2 $6.8 \%)$ to be a cause of severe bias in the data. Thus, the bias was corrected using an algorithm based on Empirical Bayes methods, as implemented in the $\mathrm{R}$ package Combat [60].

\section{Preprocessing of the gene expression microarray data}

The gene expression microarray data were preprocessed as a Lumibatch object with the lumi pipeline using $\mathrm{R}$ software [61]. Background correction was performed with the bgAdjust.affy package. The gene expression values were then transformed with vst and normalised using the rsn method. Transcripts with transformed expression values of greater than 7.5 in $20 \%$ of the samples were included in the analysis. Visualisations, boxplots and PCA plots were used in the pipeline to verify the quality of the data.

\section{Comparison of age groups}

To detect CpG sites showing substantial differences in DNA methylation between nonagenarians and young adults, the sites displaying the largest difference in the absolute value of the methylation level were included in the analysis $(-1>\Delta M>1$, threshold for $\Delta M$ based on
[61]). The rank-sums of the methylation values of the two groups were further compared with the Wilcoxon rank-sum test, and the nominal Benjamini-Hochbergadjusted p-value was set to 0.05 .

\section{Multiple regression analyses}

To assess the relationship between age- and site-specific methylation levels in greater detail, a generalised regression model referred to as variable dispersion beta regression was utilised in an iterative manner $(n=407$ 646). Age group was employed as a predictor of the site-specific methylation outcome, in the form of $\beta$ values (ranging from 0 to 1 ), in each equation of the mean model with a linker function of logit. Furthermore, as it was observed through PCA that the DNA methylation levels fluctuated based on the composition of blood cell subtypes, the proportions of CD28-/ CD4+ and CD28-/CD8+ cells showed especially clear correlations with principal component 1 , which explained $20 \%$ of the overall variance in DNA methylation. Therefore, the variables corresponding to cell type proportions (the CD4+ to $\mathrm{CD} 8+$ ratio and the proportions of CD28-/CD4+, CD28-/CD8+ and CD14+ cells) were set as adjustments in the analysis to determine leukocyte proportions independent of genome-wide ageing-associated DNA methylation changes. Sex was used as an additional covariate. The regression analyses were performed using $\mathrm{R}$ software and with algorithms implemented in the betareg package $[62,63]$. The nominal Bonferroni-adjusted p-value was set to 0.05 . See Additional file 14 for a flow chart summary of the analysis steps to identify the high-confidence ageing-associated CpG sites.

\section{Correlations with gene expression levels}

The associations between gene expression and DNA methylation levels were separately examined through bivariate correlation (Pearson) analyses for young and old individuals. The correlation analyses were designed for each transcript and $\mathrm{CpG}$ site pair showing identical annotation for a gene. Thus, multiple $\mathrm{CpG}$ sites were paired with the same gene, and several genes were matched with the same CpG site. In total, 2461 expression-methylation pairs were tested. The nominal Benjamini-Hochbergadjusted p-value was set to 0.05 .

\section{Pathway analyses}

All the pathway analyses were performed on differentially methylated genes, i.e., genes that harbour at least one ageing-associated CpG site. There were 1832 hypermethylation-associated genes (3925 CpG sites) and 2057 hypomethylation-associated genes (4615 CpG sites) included in the dataset. Of the hypomethylated CpG sites, 1719 were not associated with any known gene, and of the hypermethylated CpG sites, 720 were not associated with any known gene. 
IPA [34] was used to identify canonical pathways associated with our differentially methylated genes. According to the manufacturer, these canonical pathways are wellcharacterised metabolic and cell signalling pathways that have been curated and hand-drawn by $\mathrm{PhD}$-level scientists. All the data sources provided by the Ingenuity Knowledge Base were included in the IPA, and the Ingenuity Knowledge Base was used as the reference set in all analyses. For the association of molecules, only experimentally observed results were accepted, and only human data were considered. Benjamini-Hochberg multiple testing correction (FDR) was employed to calculate the p-values for the pathways. Canonical pathways were considered significant at $\mathrm{p}<0.05(-\log \mathrm{P}>1.3)$ and when the pathway contained a minimum of 3 genes. Pathways associated with cancer and other disease, as defined by Ingenuity Systems ${ }^{\circ}$, were excluded from the analysis. The IPA for hyper- and hypomethylation-associated genes was performed on 14.3.2014, and the IPA for genes showing a correlation between methylation and expression levels was performed on 12.3.2014.

GOrilla [31,32] was used to identify the enriched GO terms for the hyper- and hypomethylation-associated genes and for genes showing a correlation between the levels of methylation and expression. GO terms were searched based on two unranked lists (target and background), and all genes with at least one probe in the $450 \mathrm{~K}$ array were used as the background list. A Bonferronicorrected $\mathrm{p}$-value of $<0.05$ was used as the threshold for significance.

PScan [33] can be used to predict if a group of genes is regulated by a common transcription factor. The analysis was performed with the default settings, i.e., using the Jaspar database and the $-450-+50$ bp region around the TSS. PScan was able to identify 1811 and 2020 of the total hyper- and hypomethylation-associated transcripts, respectively. This analysis was performed on 11.3.2014. A Bonferroni-corrected $\mathrm{p}$-value of $<0.05$ was used as a threshold for significance.

\section{Array data}

The array data are available in the GEO database (http:// www.ncbi.nlm.nih.gov/geo/) under the accession numbers GSE40366 for the gene expression data and GSE58888 for methylation data.

\section{Additional files}

Additional file 1: All 8540 ageing-associated CpG sites.

Additional file 2: Distribution of ageing-associated methylation sites across chromosomes.

Additional file 3: Enriched GO function terms. A table of GO function terms associated with identified ageing-associated methylation sites. GO function terms associated with hypermethylated sites in sheet a and GO function terms associated with hypomethylation in sheet $b$.

Additional file 4: Diagram of enriched GO function terms. A visualisation of Additional file 3.

Additional file 5: Enriched $\mathrm{GO}$ process terms. A table of $\mathrm{GO}$ process terms associated with identified ageing-associated methylation sites. GO process terms associated with hypermethylated sites in sheet a and GO process terms associated with hypomethylation in sheet $b$.

Additional file 6: Diagram of enriched GO process terms. A visualisation of Additional file 5 .

Additional file 7: Transcription factors predicted to regulate hypermethylation-associated genes.

Additional file 8: Ingenuity canonical pathways associated with differentially methylated genes. Canonical pathways associated with hypermethylation are in sheet a and canonical pathways associated with hypomethylation are in sheet $b$.

Additional file 9: Sex- and age-group -associated methylation sites.

Additional file 10: $\mathrm{CpG}$ sites showing a correlation to the expression level of the corresponding gene.

Additional file 11: Genomic locations of the CpG sites where the level of DNA methylation correlates with the expression level of the corresponding gene.

Additional file 12: GO process terms associated with genes whose expression levels were correlating with the level of DNA methylation.

Additional file 13: PCA plots for quality control of methylation data.

Additional file 14: Flow chart of the analysis steps used to identify ageing-associated methylation sites.

\section{Abbreviations}

27K array: Infinium HumanMethylation27 BeadChip; 450K array: Infinium HumanMethylation450 BeadChip; Betareg: Beta regression model; ERV: Endogenous retrovirus; Non-CGI: Non-CpG island; PBMC: Peripheral blood mononuclear cell; TF: Transcription factor; TSS: Transcription start site; UTR: Untranslated region.

\section{Competing interests}

The authors declare they have no competing interests.

\section{Authors' contributions}

SM performed the experiments and statistical analyses and was primarily responsible for writing the manuscript. LK and SH processed the data and performed statistical analyses. JJ and TN performed the experiments. AH and $\mathrm{MJ}$ were responsible for recruiting the study population. MN performed statistical analyses. MH provided reagents and materials for the study. SM, LK, $\mathrm{JJ}$ an $\mathrm{MH}$ contributed to the design of the study. All authors contributed to the writing of the manuscript and read and approved the final manuscript.

\section{Acknowledgements}

We would like to thank Sinikka Repo-Koskinen, Janette Hinkka, Katri Välimaa and Sanna Tuominen for their skillful technical assistance.

\section{Author details}

'Department of Microbiology and Immunology, School of Medicine, University of Tampere, Tampere, Finland. ${ }^{2}$ Gerontology Research Center, Tampere, Finland. Institute of Biosciences and Medical Technology, University of Tampere, Tampere, Finland. ${ }^{4}$ School of Health Sciences, University of Tampere, Tampere, Finland. ${ }^{5}$ Fimlab Laboratories, Tampere, Finland.

Received: 11 September 2014 Accepted: 21 February 2015 Published online: 14 March 2015

References

1. López-Otín C, Blasco MA, Partridge L, Serrano M, Kroemer G. The hallmarks of aging. Cell. 2013;153:1194-217. 
2. Arnold CR, Wolf J, Brunner S, Herndler-Brandstetter D, Grubeck-Loebenstein B. Gain and loss of T cell subsets in old age - age-related reshaping of the T cell repertoire. J Clin Immunol. 2011;31:137-46.

3. Blagosklonny MV. Aging is not programmed: genetic pseudo-program is a shadow of developmental growth. Cell Cycle. 2013;12:3736-42.

4. de Magalhães JP. Programmatic features of aging originating in development: aging mechanisms beyond molecular damage? FASEB J. 2012;26:4821-6.

5. Kirkwood TB, Melov S. On the programmed/non-programmed nature of ageing within the life history. Curr Biol. 2011;21:R701-7.

6. Bacalini MG, Friso S, Olivieri F, Pirazzini C, Giuliani C, Capri M, et al. Present and future of anti-ageing epigenetic diets. Mech Ageing Dev. 2014;136:101-15.

7. Johnson AA, Akman K, Calimport SR, Wuttke D, Stolzing A, de Magalhães JP. The role of DNA methylation in aging, rejuvenation, and age-related disease. Rejuvenation Res. 2012;15:483-94.

8. Brooks-Wilson AR. Genetics of healthy aging and longevity. Hum Genet. 2013;132:1323-38

9. Riggs AD. X inactivation, differentiation, and DNA methylation. Cytogenet Cell Genet. 1975:14:9-25.

10. Holliday R, Pugh JE. DNA modification mechanisms and gene activity during development. Science. 1975;187:226-32.

11. Deaton $A M$, Bird A. CpG islands and the regulation of transcription. Genes Dev. 2011:25:1010-22.

12. Illingworth RS, Bird AP. CPG islands-'a rough guide'. FEBS Lett. 2009:583:1713-20.

13. Jones PA. Functions of DNA methylation: islands, start sites, gene bodies and beyond. Nat Rev Genet. 2012;13:484-92

14. Bogdanović O, Veenstra GJ. DNA methylation and methyl-CpG binding proteins: developmental requirements and function. Chromosoma. 2009;118:549-65.

15. Cedar H, Bergman Y. Programming of DNA methylation patterns. Annu Rev Biochem. 2012:81:97-117.

16. Christensen BC, Houseman EA, Marsit CJ, Zheng S, Wrensch MR, Wiemels J et al. Aging and environmental exposures alter tissue-specific DNA methylation dependent upon CpG island context. PLoS Genet. 2009;5:e1000602.

17. Bell JT, Tsai PC, Yang TP, Pidsley R, Nisbet J, Glass D, et al. Epigenome-wide scans identify differentially methylated regions for age and age-related phenotypes in a healthy ageing population. PLoS Gene. 2012;8:e1002629.

18. Gentilini D, Mari D, Castaldi D, Remondini D, Ogliari G, Ostan R, et al. Role of epigenetics in human aging and longevity: genome-wide DNA methylation profile in centenarians and centenarians' offspring. Age (Dordr). 2013;35:1961-73.

19. Rakyan VK, Down TA, Maslau S, Andrew T, Yang TP, Beyan H, et al. Human aging-associated DNA hypermethylation occurs preferentially at bivalent chromatin domains. Genome Res. 2010;20:434-9.

20. Teschendorff AE, Menon U, Gentry-Maharaj A, Ramus SJ, Weisenberger DJ, Shen $\mathrm{H}$, et al. Age-dependent DNA methylation of genes that are suppressed in stem cells is a hallmark of cancer. Genome Res. 2010;20:440-6.

21. Xu Z, Taylor JA. Genome-wide age-related DNA methylation changes in blood and other tissues relate to histone modification, expression and cancer. Carcinogenesis. 2014;35:356-64.

22. Weidner $\mathrm{Cl}$, Lin $\mathrm{Q}$, Koch $\mathrm{CM}$, Eisele $\mathrm{L}$, Beier F, Ziegler $\mathrm{P}$, et al. Aging of blood can be tracked by DNA methylation changes at just three CpG sites. Genome Biol. 2014;15:R24.

23. Horvath S. DNA methylation age of human tissues and cell types. Genome Biol. 2013:14:R115.

24. Steegenga WT, Boekschoten MV, Lute C, Hooiveld GJ, de Groot PJ, Morris TJ, et al. Genome-wide age-related changes in DNA methylation and gene expression in human PBMCs. Age (Dordr). 2014;36:9648.

25. Florath I, Butterbach K, Muller H, Bewerunge-Hudler M, Brenner H. Cross-sectional and longitudinal changes in DNA methylation with age: an epigenome-wide analysis revealing over 60 novel age-associated $\mathrm{CpG}$ sites. Hum Mol Genet. 2013:23:1186-201.

26. Garagnani P, Bacalini MG, Pirazzini C, Gori D, Giuliani C, Mari D, et al. Methylation of ELOVL2 gene as a new epigenetic marker of age. Aging Cell. 2012;11:1132-4.

27. Hannum G, Guinney J, Zhao L, Zhang L, Hughes G, Sadda S, et al. Genome-wide methylation profiles reveal quantitative views of human aging rates. Mol Cell. 2012;49:359-67.

28. Heyn H, Li N, Ferreira HJ, Moran S, Pisano DG, Gomez A, et al. Distinct DNA methylomes of newborns and centenarians. Proc Natl Acad Sci U S A. 2012;109:10522-7.
29. Jaffe AE, Irizarry RA. Accounting for cellular heterogeneity is critical in epigenome-wide association studies. Genome Biol. 2014;15:R31.

30. Marttila S, Jylhävä J, Pesu M, Hämäläinen S, Jylhä M, Hervonen A, et al. IL-7 concentration is increased in nonagenarians but is not associated with markers of T cell immunosenescence. Exp Gerontol. 2011;46:1000-2.

31. Eden E, Lipson D, Yogev S, Yakhini Z. Discovering motifs in ranked lists of DNA sequences. PLoS Comput Biol. 2007;3:e39.

32. Eden $E$, Navon $R$, Steinfeld I, Lipson D, Yakhini Z. GOrilla: a tool for discovery and visualization of enriched $\mathrm{GO}$ terms in ranked gene lists. BMC Bioinformatics. 2009:10:48.

33. Zambelli F, Pesole G, Pavesi G. Pscan: finding over-represented transcription factor binding site motifs in sequences from co-regulated or co-expressed genes. Nucleic Acids Res. 2009;37:W247-52.

34. Ingenuity Systems ${ }^{\circledast}$, www.ingenuity.com.

35. Marttila S, Jylhävä J, Nevalainen $T$, Nykter $M$, Jylhä $M$, Hervonen A, et al. Transcriptional analysis reveals gender-specific changes in the aging of the human immune system. PLoS One. 2013;8:e66229.

36. Johansson A, Enroth S, Gyllensten U. Continuous aging of the human DNA methylome throughout the human lifespan. PLoS One. 2013;8:e67378

37. McClay JL, Aberg KA, Clark SL, Nerella S, Kumar G, Xie LY, et al. A methylome-wide study of aging using massively parallel sequencing of the methyl-CpG-enriched genomic fraction from blood in over 700 subjects. Hum Mol Genet. 2014;23:1175-85.

38. D'Aquila P, Rose G, Bellizzi D, Passarino G. Epigenetics and aging. Maturitas, 2013;74:130-6.

39. Pawelec G, Larbi A, Derhovanessian E. Senescence of the human immune system. J Comp Pathol. 2010;142 Suppl 1:S39-44.

40. Zilbauer M, Rayner TF, Clark C, Coffey AJ, Joyce CJ, Palta P, et al. Genome-wide methylation analyses of primary human leukocyte subsets identifies functionally important cell-type-specific hypomethylated regions. Blood. 2013;122:e52-60

41. Kondilis-Mangum HD, Wade PA. Epigenetics and the adaptive immune response. Mol Aspects Med. 2013:34:813-25.

42. Lam LL, Emberly E, Fraser HB, Neumann SM, Chen E, Miller GE, et al. Factors underlying variable DNA methylation in a human community cohort. Proc Natl Acad Sci U S A. 2012;109 Suppl 2:17253-60.

43. Lleras RA, Adrien LR, Smith RV, Brown B, Jivraj N, Keller C, et al. Hypermethylation of a cluster of Krüppel-type zinc finger protein genes on chromosome 19q13 in oropharyngeal squamous cell carcinoma. Am J Pathol. 2011;178:1965-74.

44. Lukic S, Nicolas JC, Levine AJ. The diversity of zinc-finger genes on human chromosome 19 provides an evolutionary mechanism for defense against inherited endogenous retroviruses. Cell Death Differ. 2014;21:381-7

45. Gaubatz JW, Arcement B, Cutler RG. Gene expression of an endogenous retrovirus-like element during murine development and aging. Mech Ageing Dev. 1991;57:71-85.

46. Wada Y, Tsukada M, Kamiyama S, Koizumi A. Retroviral gene expression as a possible biomarker of aging. Int Arch Occup Environ Health. 1993;65(1 Suppl):S235-9.

47. Romanish MT, Cohen CJ, Mager DL. Potential mechanisms of endogenous retroviral-mediated genomic instability in human cancer. Semin Cancer Biol. 2010;20:246-53.

48. Sharif $R$, Thomas $P$, Zalewski $P$, Fenech $M$. The role of zinc in genomic stability. Mutat Res. 2012;733:111-21.

49. Mocchegiani E, Costarelli L, Giacconi R, Cipriano C, Muti E, Malavolta M. Zinc-binding proteins (metallothionein and alpha-2 macroglobulin) and immunosenescence. Exp Gerontol. 2006;41:1094-107.

50. Maunakea AK, Nagarajan RP, Bilenky M, Ballinger TJ, D'Souza C, Fouse SD, et al. Conserved role of intragenic DNA methylation in regulating alternative promoters. Nature. 2010:466:253-7.

51. Fenteany G, Glogauer M. Cytoskeletal remodeling in leukocyte function. Curr Opin Hematol. 2004;11:15-24.

52. Zykovich A, Hubbard A, Flynn JM, Tarnopolsky M, Fraga MF, Kerksick C, et al. Genome-wide DNA methylation changes with age in disease-free human skeletal muscle. Aging Cell. 2014;13:360-6.

53. Bhutani N, Burns DM, Blau HM. DNA demethylation dynamics. Cell. 2011;146:866-72.

54. Jones PA, Liang G. Rethinking how DNA methylation patterns are maintained. Nat Rev Genet. 2009;10:805-11. 
55. Bellizzi D, D'Aquila P, Montesanto A, Corsonello A, Mari V, Mazzei B, et al. Global DNA methylation in old subjects is correlated with frailty. Age (Dordr). 2012;34:169-79.

56. Goebeler S, Jylhä M, Hervonen A. Medical history, cognitive status and mobility at the age of 90. A population based study in Tampere, Finland. Aging Clin Exp Res. 2003;15:154-61.

57. Pidsley R, Wong CC Y, Volta M, Lunnon K, Mill J, Schalkwyk LC. A data-driven approach to preprocessing Illumina 450K methylation array data. BMC Genomics. 2013;14:293.

58. Chen YA, Lemire M, Choufani S, Butcher DT, Grafodatskaya D, Zanke BW, et al. Discovery of cross-reactive probes and polymorphic CpGs in the Illumina Infinium HumanMethylation450 microarray. Epigenetics. 2013;8:203-9.

59. Teschendorff AE, Marabita F, Lechner M, Bartlett T, Tegner J, Gomez-Cabrero $D$, et al. A beta-mixture quantile normalization method for correcting probe design bias in Illumina Infinium 450 k DNA methylation data. Bioinformatics. 2013;29:189-96.

60. Johnson WE, Li C, Rabinovic A. Adjusting batch effects in microarray expression data using empirical Bayes methods. Biostatistics. 2007:8:118-27.

61. Du P, Kibbe WA, Lin SM. lumi: a pipeline for processing Illumina microarray. Bioinformatics. 2008;24:1547-8.

62. Cribari-Neto F, Zeileis A. Beta regression in R. JSS. 2010;34:1-24.

63. Ferrari C. Beta regression for modelling rates and proportions. J Appl Stat. 2004;31:799-15.

\section{Submit your next manuscript to BioMed Central and take full advantage of:}

- Convenient online submission

- Thorough peer review

- No space constraints or color figure charges

- Immediate publication on acceptance

- Inclusion in PubMed, CAS, Scopus and Google Scholar

- Research which is freely available for redistribution 